\title{
Numerical Green's Function Method Based on the DE Transformation
}

\author{
Masatake Mori and Toshihiko Echigo \\ Department of Mathematical Sciences, Tokyo Denki University, \\ Hatoyama, Hiki-gun, Saitama 350-0394, Japan
}

Received September 2, 2005

Revised January 16, 2006

\begin{abstract}
A method for numerical solution of boundary value problems with ordinary differential equation based on the method of Green's function incorporated with the double exponential transformation is presented. The method proposed does not require solving a system of linear equations and gives an approximate solution of very high accuracy with a small number of function evaluations. The error of the method is $O\left(\exp \left(-C_{1} N / \log \left(C_{2} N\right)\right)\right)$ where $N$ is a parameter representing the number of function evaluations and $C_{1}$ and $C_{2}$ are some positive constants. Numerical examples also prove the high efficiency of the method. An alternative method via an integral equation is presented which can be used when the Green's function corresponding to the given equation is not available.
\end{abstract}

Key words: double exponential transformation, DE transformation, Green's function, boundary value problem, integral equation

\section{Introduction}

There have been several methods for numerical solution of boundary value problems with ordinary differential equation. The shooting method is a traditional one and the implicit finite difference method is also one of the useful tools. In 2002 Sugihara [8] and in 2005 Nurmuhammad et al. [5] proposed sinc collocation methods based on the double exponential transformation. In the present paper, a method for numerical solution of boundary value problems based on the classical method of Green's function incorporated with the double exponential transformation is presented. Although the method of Green's function is a classical one for analytical manipulation of solution of boundary value problems with differential equation, the method presented here gives an approximate solution of very high accuracy with a small number of function evaluations.

The differential equation we consider here is

$$
L[u]+f(x)=0, \quad a<x<b,
$$

where $u(x)$ is the function to be determined and $L[u]$ is a self-adjoint operator associated with the Sturm-Liouville eigen-value problem defined as

$$
L[u] \equiv \frac{d}{d x}\left\{p(x) \frac{d u}{d x}\right\}+q(x) u .
$$

We assume here that $p(x), q(x)$ and $f(x)$ are known and analytic on $(a, b)$, and $f(x)$ may be singular at $x=a, b$ provided that the equation is defined properly. We also 
assume that $p(x)>0$ on $(a, b)$. For the moment we assume that the boundary condition is homogeneous, i.e.

$$
u(a)=0, \quad u(b)=0 .
$$

However, if $p(x)$ vanishes at $x=a$ or $x=b$ we can impose there an inhomogeneous boundary condition

$$
u(a)=\text { finite } \neq 0, \quad \text { or } \quad u(b)=\text { finite } \neq 0
$$

as will be shown later in Example 2.

\section{The Method of Green's Function}

We start with a short revisit to the method of Green's function [1]. We consider here the following boundary value problem with homogeneous differential equation with the same $L[u]$ as given in (1.2):

$$
\left\{\begin{array}{l}
L[y]=0 \\
y(a)=0, \quad y(b)=0 .
\end{array}\right.
$$

It is well known that the Green's function $K(x, \xi)$ corresponding to the operator $L[y]$ in (2.1) with the homogeneous boundary condition is defined as follows:

1. For fixed $\xi, K(x, \xi)$ is a continuous function of $x$ and satisfies the homogeneous boundary condition (1.3).

2. The first and the second derivatives of $K(x, \xi)$ with respect to $x$ are continuous on $(a, b)$ except $x=\xi$ and $d K / d x$ satisfies

$$
\left(\frac{d K}{d x}\right)_{x=\xi+0}-\left(\frac{d K}{d x}\right)_{x=\xi-0}=-\frac{1}{p(\xi)} .
$$

3. $K(x, \xi)$ satisfies the differential equation $(2.1)$ on $(a, b)$ except $x=\xi$ as a function of $x$.

Let $y_{1}$ and $y_{2}$ be fundamental solutions of $L(y)=0$ which satisfy $y_{1}(a)=0$ and $y_{2}(b)=0$, respectively. Then the Green's function can be written

$$
K(x, \xi)=\left\{\begin{array}{cc}
y_{1}(\xi) y_{2}(x), & \xi \leq x \\
y_{1}(x) y_{2}(\xi), & x \leq \xi
\end{array}\right.
$$

Under these preliminaries the solution of the boundary value problem of inhomogeneous equation (1.1) is expressed as follows [1]:

$$
u(x)=\int_{a}^{b} K(x, \xi) f(\xi) d \xi
$$




\section{The DE Formula for Indefinite Integrals}

The derivative of the integrand in (2.4) has a discontinuity at $\xi=x$ and we can not apply the double exponential formula for definite integrals over $(a, b)$ to (2.4) [10]. However, we can write (2.4) in terms of a sum of two indefinite integrals with an analytic integrand over each interval of integration:

$$
u(x)=\int_{a}^{x} K(x, \xi) f(\xi) d \xi+\int_{x}^{b} K(x, \xi) f(\xi) d \xi .
$$

And hence it is quite natural that we employ a numerical integrator that gives a good result for each of the indefinite integral whose integrand is regular on each interval.

In 2003 Muhammad and Mori [3] and in 2004 Tanaka, Sugihara and Murota [11] proposed a double exponential formula for indefinite integral which works well for such kind of integrals. We first consider here an indefinite integral of a function $g(\xi)$ over $(a, x)$ corresponding to the first term in the right-hand side of $(3.1)$ :

$$
I_{1}(x)=\int_{a}^{x} g(\xi) d \xi, \quad a<x<b .
$$

Here we assume that $g(\xi)$ is regular over $(a, b)$ except $\xi=a$ and $b$. If we apply the variable transformation

$$
\xi=\psi(t)=\frac{b-a}{2} \tanh \left(\frac{\pi}{2} \sinh t\right)+\frac{b+a}{2}
$$

to $(3.2)$ we have

$$
I_{1}(x)=\int_{-\infty}^{\tau} g(\psi(t)) \psi^{\prime}(t) d t, \quad \tau=\psi^{-1}(x)
$$

We assume here that $g(\psi(t)) \psi^{\prime}(t)$ satisfies

$$
g(\psi(t)) \psi^{\prime}(t) \text { is regular in the strip }|\operatorname{Im} t|<d \text { for some } d>0
$$

and

$$
g(x)=O\left(((x-a)(b-x))^{-1+\alpha}\right) \quad \text { as } x \rightarrow a, b
$$

with some positive constant $\alpha$. Transformation (3.3) is first proposed by Takahasi and Mori in 1974 [10] for numerical definite integration and is called the double exponential transformation, abbreviated as the DE transformation, because the integrand $g(\psi(t)) \psi^{\prime}(t)$ after the transformation decays double exponentially, i.e.,

$$
g(\psi(t)) \psi^{\prime}(t)=O\left(\exp \left(-\frac{\pi(\alpha-\varepsilon)}{2} \exp |t|\right)\right), \quad \text { as } t \rightarrow \pm \infty
$$

for an arbitrarily small positive number $\varepsilon$. 
Under the assumptions given above with some additional mild ones $g(\psi(t)) \psi^{\prime}(t)$ can be expanded in terms of the sinc function $[9,6,3]$ with mesh size $h$ :

$$
\begin{aligned}
g(\psi(t)) \psi^{\prime}(t) & =\sum_{j=-\infty}^{\infty} g(\psi(j h)) \psi^{\prime}(j h) \operatorname{sinc}\left(\frac{t}{h}-j\right)+\lim _{n \rightarrow \infty} e_{n} \\
e_{n} & =\frac{\sin (\pi t / h)}{2 \pi i} \int_{C_{n}} \frac{g(\psi(\tau)) \psi^{\prime}(\tau)}{(\tau-t) \sin (\pi \tau / h)} d \tau
\end{aligned}
$$

where the sinc function $\operatorname{sinc}(t)$ is defined

$$
\operatorname{sinc}(t)=\frac{\sin \pi t}{\pi t}
$$

and $\lim _{n \rightarrow \infty} e_{n}$ is the error term in which the path $C_{n}$ surrounds all the simple poles $\tau=j h, j=0, \pm 1, \ldots, \pm n$ and $\tau=t$ in the positive direction but not the singularities of $g(\psi(\tau)) \psi^{\prime}(\tau)$. We substitute (3.8) into (3.4) and truncate the infinite summation at $\pm N$ in such a way that

$$
N=\frac{1}{h} \log \frac{2 d}{\alpha^{\prime} h}, \quad \alpha^{\prime}=\alpha-\varepsilon
$$

holds. Relation (3.10) comes from the requirement that the discretization error $\lim _{n \rightarrow \infty} e_{n}$ due to the sinc expansion and the error due to the truncation of the infinite summation (3.8) be the same order of magnitude. Then we have the double exponential formula, abbreviated as the DE formula, for indefinite integral [3]

$$
I_{1}(x)=h \sum_{j=-N}^{N} g(\psi(j h)) \psi^{\prime}(j h)\left(\frac{1}{2}+\frac{1}{\pi} \operatorname{Si}\left(\pi \frac{\psi^{-1}(x)}{h}-\pi j\right)\right)+E_{N 1},
$$

where $\operatorname{Si}(t)$ is the sine integral defined by

$$
\operatorname{Si}(t)=\int_{0}^{t} \frac{\sin \tau}{\tau} d \tau
$$

and $E_{N 1}$ is the error term given by

$$
E_{N 1}=O\left(\exp \left(-\frac{\pi d N}{\log \left(2 d N / \alpha^{\prime}\right)}\right)\right)
$$

which shows a nearly exponential decay of the errror of the DE formula in terms of $N$. Here we first gave $h$ and then determined $N$ by (3.10). However, if we want to give first $N$ then to determine $h$, it should be determined

$$
h=\frac{1}{N} \log \frac{2 d N}{\alpha^{\prime}}, \quad \alpha^{\prime}=\alpha-\varepsilon .
$$

Although in (3.10) and (3.14) $\varepsilon$ need to be an arbitrarily small positive number from the theoretical view point, in actual computation we can take $\varepsilon=0$. 
Next we consider the indefinite integral of $g(\xi)$ over $(x, b)$ corresponding to the second term of the right-hand side of (3.1):

$$
I_{2}(x)=\int_{x}^{b} g(\xi) d \xi, \quad a<x<b .
$$

Completely in the same way as in $I_{1}(x)$ we obtain the DE formula for indefinite integral (3.15):

$$
I_{2}(x)=h \sum_{j=-N}^{N} g(\psi(j h)) \psi^{\prime}(j h)\left(\frac{1}{2}-\frac{1}{\pi} \operatorname{Si}\left(\pi \frac{\psi^{-1}(x)}{h}-\pi j\right)\right)+E_{N 2},
$$

where $E_{N 2}$ is the same as $E_{N 1}$ in (3.13). Note that the only difference between (3.11) and (3.16) is the sign of the term $\mathrm{Si} / \pi$.

\section{The DE Formula for the Method of Green's Function}

Now we return to the method of Green's function. The integral we want to evaluate is $(3.1)$, i.e.

$$
\begin{aligned}
u(x) & =\int_{a}^{x} K(x, \xi) f(\xi) d \xi+\int_{x}^{b} K(x, \xi) f(\xi) d \xi \\
& =J_{1}(x)+J_{2}(x)
\end{aligned}
$$

where

$$
\begin{aligned}
& J_{1}(x)=y_{2}(x) \int_{a}^{x} y_{1}(\xi) f(\xi) d \xi \\
& J_{2}(x)=y_{1}(x) \int_{x}^{b} y_{2}(\xi) f(\xi) d \xi
\end{aligned}
$$

from (2.3). If we approximate $J_{1}(x)$ and $J_{2}(x)$ by (3.11) and (3.16), respectively, we immediately have

$$
\begin{aligned}
u(x)= & y_{2}(x) h \sum_{j=-N}^{N} y_{1}(\psi(j h)) f(\psi(j h)) \psi^{\prime}(j h)\left(\frac{1}{2}+\frac{1}{\pi} \operatorname{Si}\left(\pi \frac{\psi^{-1}(x)}{h}-\pi j\right)\right) \\
& +y_{1}(x) h \sum_{j=-N}^{N} y_{2}(\psi(j h)) f(\psi(j h)) \psi^{\prime}(j h)\left(\frac{1}{2}-\frac{1}{\pi} \operatorname{Si}\left(\pi \frac{\psi^{-1}(x)}{h}-\pi j\right)\right) \\
& +E_{N},
\end{aligned}
$$

where $E_{N}$ is the same as $E_{N 1}$ in (3.13). This is the DE formula for numerical solution of the boundary value problem (1.1) with homogeneous boundary condition (1.3). Since we can compute $y_{1}(\psi(j h)) f(\psi(j h)) \psi^{\prime}(j h)$ and $y_{2}(\psi(j h)) f(\psi(j h)) \psi^{\prime}(j h)$ 
beforehand for each $j$, what we have only to compute for a given $x$ is $y_{1}(x), y_{2}(x)$ and $1 / 2 \pm \operatorname{Si}\left(\pi \psi^{-1}(x) / h-\pi j\right) / \pi$ and their product sum. Thus, this formula for $x$ consists of evaluations of simple functions and their sum, so that the present method is suitable for parallel computation. Also note that if $x$ is equal to one of the sinc points, i.e., $x=\psi(k h)$ for some integer $k$,

$$
\operatorname{Si}\left(\pi \psi^{-1}(x) / h-\pi j\right)=\operatorname{Si}(\pi(k-j))
$$

holds and computation of Si becomes very simple.

\section{Numerical Examples}

In this section we give some numerical examples. The interval of definition of the problems are $(0,1)$ in all the examples. For each example we first chose $N=4,8,16,32, \ldots$ and computed $h$ by $(3.14)$. For each $N$ we evaluated $u(x)$ by means of the formula (4.4) for

$$
x=0.01,0.02,0.03, \ldots, 0.97,0.98,0.99
$$

and picked up the maximum absolute value of the error. Since in the integrand of (4.4) the singular points which lie nearest to the real axis are the poles of $\psi^{\prime}(t)=$ $\frac{\pi}{4} \cosh t / \cosh ^{2}(\pi / 2 \sinh t)$ with the distance $\pi / 2$ [10], we set $d=\pi / 2$ in all the examples. In order to show the high efficiency of the present formula we carried out numerical computation with quadruple precision arithmetic.

Example 1.

$$
\left\{\begin{array}{l}
\frac{d^{2} u}{d x^{2}}-\frac{3}{4}\left(x^{-\frac{1}{2}}+(1-x)^{-\frac{1}{2}}\right)=0 \\
u(0)=0, \quad u(1)=0
\end{array}\right.
$$

Here we take

$$
L[u]=\frac{d^{2} u}{d x^{2}}
$$

and the Green's function corresponding to (5.3) is

$$
y_{1}(x)=x, \quad y_{2}(x)=1-x,
$$

i.e.,

$$
K(x, \xi)= \begin{cases}\xi(1-x), & \xi \leq x \\ x(1-\xi), & x \leq \xi\end{cases}
$$

The exact solution of this problem is

$$
u(x)=x^{\frac{3}{2}}+(1-x)^{\frac{3}{2}}-1 .
$$


In this example, as $\xi$ tends to $0 f(\xi)=-3 / 4\left(\xi^{-1 / 2}+(1-\xi)^{-1 / 2}\right)=O\left(\xi^{-1 / 2}\right)$ from $(5.2)$ and $y_{1}(\xi)=O(\xi)$ from $(5.4)$, so that $g(\xi)=y_{2}(x) f(\xi) y_{1}(\xi)=O\left(\xi^{1 / 2}\right)=$ $O\left(\xi^{-1+\alpha}\right), \alpha=3 / 2$ holds. In a similar way we see that as $\xi$ tends to $1 g(\xi)=$ $O\left((1-\xi)^{1 / 2}\right)$. In this way we chose $\alpha=3 / 2$ for (3.6) in this example. In other examples we determined $\alpha$ in the same way.

We chose $N=4,8,16,32,64,76$, computed numerical solution for $x$ 's given in (5.1) and plotted the maximum absolute error of the numerical solution as a function of $N$ in Fig. 1. Although $f(x)$ of this problem is singular at $x=0$ and 1 , the result is very good. Actually, the error decays almost exponentially as given in (3.13) and attains about $10^{-30}$ with $N=76$.

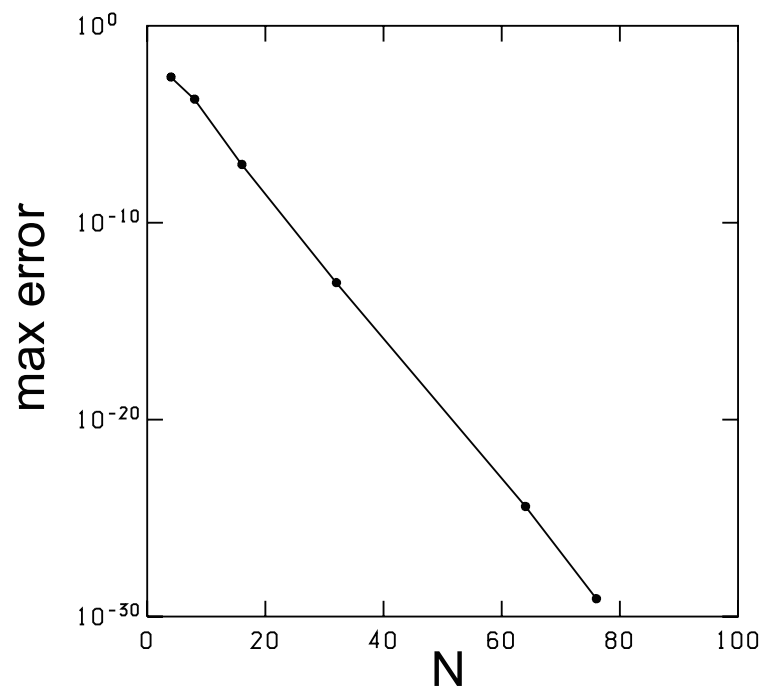

Fig. 1. The maximum error of Example 1.

Example 2.

In this example we take

$$
\left\{\begin{array}{l}
x \frac{d^{2} u}{d x^{2}}+\frac{d u}{d x}+x=0 \\
u(0)=\text { finite, } \quad u(1)=0 .
\end{array}\right.
$$

$$
L[u]=x \frac{d^{2} u}{d x^{2}}+\frac{d u}{d x}=\frac{d}{d x}\left(x \frac{d u}{d x}\right) .
$$

The Green's function corresponding to (5.8) is

$$
y_{1}(x)=1, \quad y_{2}(x)=-\log x,
$$

i.e.,

$$
K(x, \xi)= \begin{cases}-\log x, & \xi \leq x \\ -\log \xi, & x \leq \xi\end{cases}
$$


The exact solution of this problem is

$$
u(x)=\frac{1}{4}\left(1-x^{2}\right) .
$$

In this example $p(x)=x$ in (1.2) and it vanishes at $x=0$, so that we can impose a boundary condition $u(0)=$ finite $\neq 0$. Actually we see from $(5.11)$ that $u(0)=1 / 4$. We take $\alpha=2$ in this example. We chose $N=4,8,16,32,64,76$, computed numerical solution for $x$ 's given in (5.1) and plotted the maximum absolute error as a function of $N$ in Fig. 2. In this example also the error behavior given by (3.13) is observed.

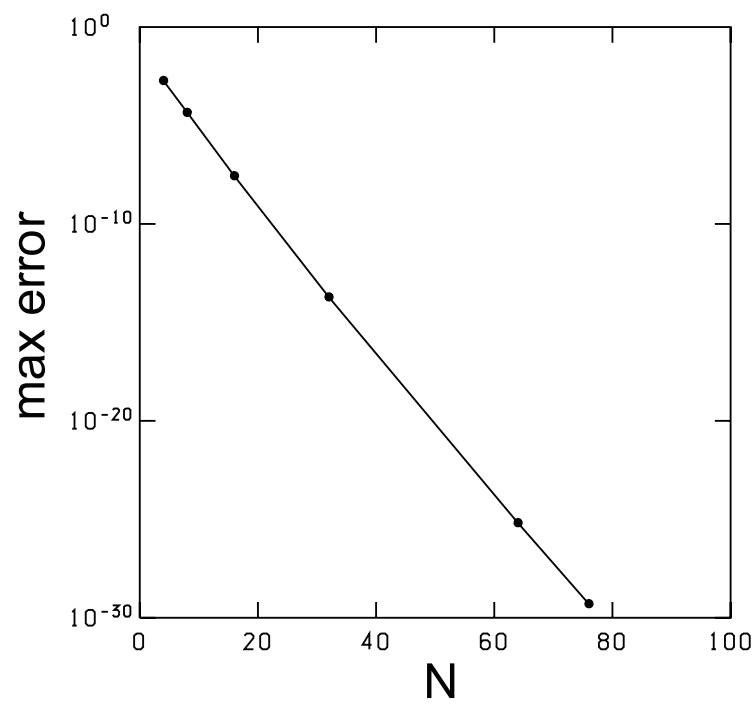

Fig. 2. The maximum error of Example 2.

Example 3. Next example is from [7, p. 550]:

$$
\left\{\begin{array}{l}
\frac{d^{2} u}{d x^{2}}-\nu^{2} u-\nu^{2} \cos ^{2} \pi x-2 \pi^{2} \cos 2 \pi x=0, \\
u(0)=0, \quad u(1)=0 .
\end{array}\right.
$$

Although the equation with $\nu=20$ is considered in [7] we compute here numerical solutions with $\nu=1, \nu=10$ and $\nu=20$, and compare the accuracy. In this example we take

$$
L[u]=\frac{d^{2} u}{d x^{2}}-\nu^{2} u .
$$

The Green's function corresponding to (5.13) is

$$
y_{1}(x)=\frac{\sinh \nu x}{\nu \sinh \nu}, \quad y_{2}(x)=\sinh \nu(1-x),
$$


i.e.,

$$
K(x, \xi)= \begin{cases}\frac{\sinh \nu \xi \sinh \nu(1-x)}{\nu \sinh \nu}, & \xi \leq x \\ \frac{\sinh \nu(1-\xi) \sinh \nu x}{\nu \sinh \nu}, & x \leq \xi\end{cases}
$$

The exact solution of this problem is

$$
u(x)=\frac{\exp (-\nu)}{1+\exp (-\nu)} \exp (\nu x)+\frac{1}{1+\exp (-\nu)} \exp (-\nu x)-\cos ^{2} \pi x .
$$

We take $\alpha=2$ in this example. We chose $N=4,8,16,32,64,128$, computed numerical solution for $x$ 's given in (5.1) and plotted the maximum absolute error as a function of $N$ in Fig. 3.

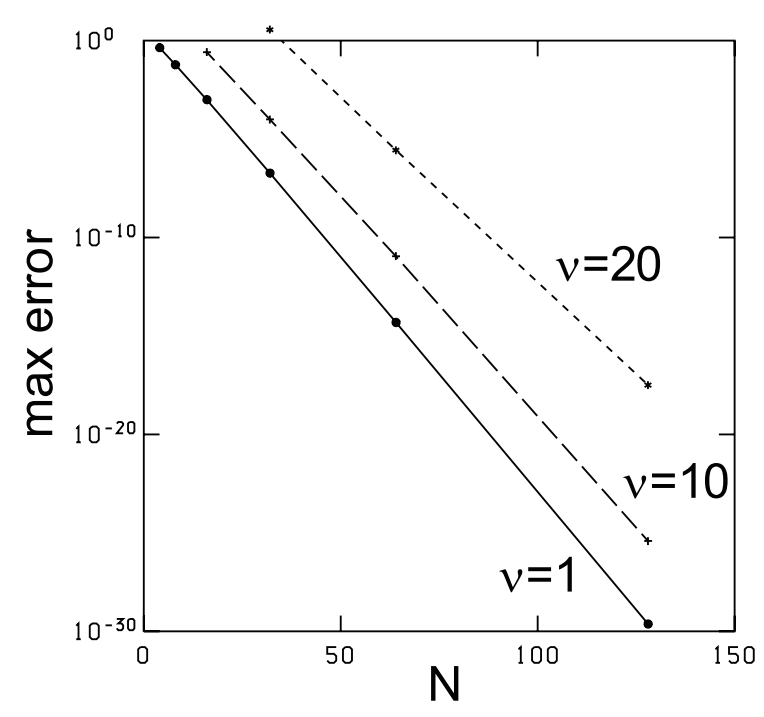

Fig. 3. The maximum error of Example 3.

If we try to solve this equation with $\nu=20$ by means of one of the conventional difference methods we usually find it difficult to obtain a good solution because of a sharp growth and a sharp decay of the solution due to the latent factors $\exp (\nu x)$ and $\exp (-\nu x)$ in (5.16). In the present method, on the other hand, while with small $N$ we fail to obtain a good result, we observe in Fig. 3 an exponential rate of convergence of the error expressed as (3.13) as $N$ becomes large. For smaller $\nu$ the situation is much better. See the error curves corresponding to $\nu=10$ and $\nu=1$ in Fig. 3.

The reason why the present method fails to obtain a good result when $\nu$ is large and $N$ is small can be explained as follows. First note that the support of the function $1 / 2+\operatorname{Si}\left(\pi \psi^{-1}(x) / h-\pi j\right) / \pi$ in $(3.11)$ is the entire interval $(a, b)$, although the amplitude of $1 / 2+\operatorname{Si}\left(\pi \psi^{-1}(x) / h-\pi j\right) / \pi$ is small if $x<\psi(j h)$ [3]. And hence 
the formula for indefinite integration (3.11) samples values not only from inside the interval $(a, x)$ but also from outside the interval $(a, x)$. This is one of the significant characteristics of the formula (3.11). As for (4.4), the first term in the right-hand side samples the values of $y_{1}(\psi(j h))$ even when $\psi^{-1}(x) / h<j$. If $\nu$ is large, while the shape of the Green's function $K(x, \xi)$ is very sharp, $y_{1}(\psi(j h))$ becomes quite large for large $j$, although $\psi^{\prime}(j h)$ and other factors lower the effect of $y_{1}(\psi(j h))$ to some extent. Thus, when $\nu$ is large and $N$ is small, the major part of the sample points are located outside the effective support of the Green's function and the distribution of the sample points does not follow properly the sharp peak of the Green's function, which deteriorates the accuracy of the formula. Situation is the same for the second term in the right-hand side of (4.4). As $N$ becomes large the distribution of the sample points comes to follow properly the shape of the Green's function and the accuracy changes for the better quickly.

\section{Integral Equation}

It is not always possible to find a relevant Green's function corresponding to the given differential operator. In such a case we separate the given operator into a sum of $L[u]$ whose Green's function is known and other terms including $u$. In this section we consider a boundary value problem with a linear differential equation

$$
\left\{\begin{array}{l}
L[u]+\rho(x) u+f(x)=0 \\
u(a)=0, \quad u(b)=0
\end{array}\right.
$$

where the Green's function corresponding to $L[u]$ is known but not known corresponding to $L[u]+\rho(x) u$. If we regard $\rho(x) u+f(x)$ as an inhomogeneous term we see from (2.4) that the equation (6.1) can be transformed into the following equivalent integral equation:

$$
u(x)=\int_{a}^{b} K(x, \xi)(\rho(\xi) u(\xi)+f(\xi)) d \xi
$$

This equation seems to be formally a Fredholm integral equation. However, as seen from (3.1) we should regard (6.2) as a sum of two Volterra integral equations, i.e. we should divide the integral over $(a, b)$ into the integral over $(a, x)$ and the other one over $(x, b)$ :

$$
u(x)=\int_{a}^{x} K(x, \xi)(\rho(\xi) u(\xi)+f(\xi)) d \xi+\int_{x}^{b} K(x, \xi)(\rho(\xi) u(\xi)+f(\xi)) d \xi
$$

In order to solve this integral equation numerically we employ the method proposed by Muhammad et al. [4]. Incidentally we should note here that a general theory of the method of Green's function based on quadrature is developed as integral equations methods in the book by H. B. Keller $[2]^{1}$. Now, first we apply the DE

\footnotetext{
${ }^{1}$ The authors are indebted to the referee for bringing this book to their notice.
} 
formula (4.4) to the right-hand side and have

$$
\begin{aligned}
u(x)=y_{2}(x) h \sum_{j=-N}^{N} y_{1}(\psi(j h))( & \rho(\psi(j h)) u(\psi(j h))+f(\psi(j h))) \\
& \times \psi^{\prime}(j h)\left(\frac{1}{2}+\frac{1}{\pi} \operatorname{Si}\left(\pi \frac{\psi^{-1}(x)}{h}-\pi j\right)\right) \\
+ & y_{1}(x) h \sum_{j=-N}^{N} y_{2}(\psi(j h))(\rho(\psi(j h)) u(\psi(j h))+f(\psi(j h))) \\
& \times \psi^{\prime}(j h)\left(\frac{1}{2}-\frac{1}{\pi} \operatorname{Si}\left(\pi \frac{\psi^{-1}(x)}{h}-\pi j\right)\right) \\
+E_{N} . &
\end{aligned}
$$

Then, in order to derive an equation whose solution gives an approximation to $u\left(x_{j}\right), j=0, \pm 1, \ldots, \pm N$ we apply the collocation method based on the sinc points $x=\psi(k h), k=0, \pm 1, \pm 2, \ldots, \pm N$ and obtain [4]

$$
\begin{gathered}
u_{k}=y_{2}(\psi(k h)) h \sum_{j=-N}^{N} y_{1}(\psi(j h))\left(\rho(\psi(j h)) u_{j}+f(\psi(j h))\right) \\
\times \psi^{\prime}(j h)\left(\frac{1}{2}+\frac{1}{\pi} \operatorname{Si}(\pi(k-j))\right) \\
+y_{1}(\psi(k h)) h \sum_{j=-N}^{N} y_{2}(\psi(j h))\left(\rho(\psi(j h)) u_{j}+f(\psi(j h))\right) \\
\times \psi^{\prime}(j h)\left(\frac{1}{2}-\frac{1}{\pi} \operatorname{Si}(\pi(k-j))\right), \\
k=0, \pm 1, \pm 2, \ldots, \pm N
\end{gathered}
$$

where $u_{i}$ is an approximate value to the solution $u(x)$ at the sinc point $x=\psi(i h)$. Here we used the relation (4.5). This equation can be written in a matrix form:

$$
(I-h M) \boldsymbol{u}=h \boldsymbol{b},
$$

where $I$ is the identity matrix and

$$
\begin{aligned}
M_{k j}= & y_{2}(\psi(k h)) y_{1}(\psi(j h)) \rho(\psi(j h)) \psi^{\prime}(j h)\left(\frac{1}{2}+\frac{1}{\pi} \operatorname{Si}(\pi(k-j))\right) \\
& +y_{1}(\psi(k h)) y_{2}(\psi(j h)) \rho(\psi(j h)) \psi^{\prime}(j h)\left(\frac{1}{2}-\frac{1}{\pi} \operatorname{Si}(\pi(k-j))\right), \\
\boldsymbol{u}= & \left(u_{-N}, u_{-N+1}, \ldots, u_{N-1}, u_{N}\right)^{\mathrm{T}}, \\
\boldsymbol{b}= & \left(b_{-N}, b_{-N+1}, \ldots, b_{N-1}, b_{N}\right)^{\mathrm{T}},
\end{aligned}
$$




$$
\begin{aligned}
b_{k}= & y_{2}(\psi(k h)) \sum_{j=-N}^{N} y_{1}(\psi(j h)) f(\psi(j h)) \psi^{\prime}(j h)\left(\frac{1}{2}+\frac{1}{\pi} \operatorname{Si}(\pi(k-j))\right) \\
& +y_{1}(\psi(k h)) \sum_{j=-N}^{N} y_{2}(\psi(j h)) f(\psi(j h)) \psi^{\prime}(j h)\left(\frac{1}{2}-\frac{1}{\pi} \operatorname{Si}(\pi(k-j))\right) .
\end{aligned}
$$

This is a linear system of algebraic equations with respect to $u_{j}$ 's and we can usually solve it.

If we want to get an approximate value of the solution at an arbitrary $x$ we can compute it by $(6.4)$ without $E_{n}$ in which $u(\psi(j h))$ is replaced by $u_{j}$.

Example 4. We again consider the problem (5.12), i.e.,

$$
\left\{\begin{array}{l}
\frac{d^{2} u}{d x^{2}}-\nu^{2} u-\nu^{2} \cos ^{2} \pi x-2 \pi^{2} \cos 2 \pi x=0 \\
u(0)=0, \quad u(1)=0
\end{array}\right.
$$

in which we take here

$$
L[u]=\frac{d^{2} u}{d x^{2}}
$$

and hence the Green's function is given by (5.5). We choose $\nu=1$ and $\nu=20$ and assign the same values to $\alpha, N$ and $h$ as those given in Example 3. We solved algebraic equation (6.6) using the LU decomposition. We evaluated approximate solution for the same $x$ 's in (5.1) and plotted the maximum absolute error as a

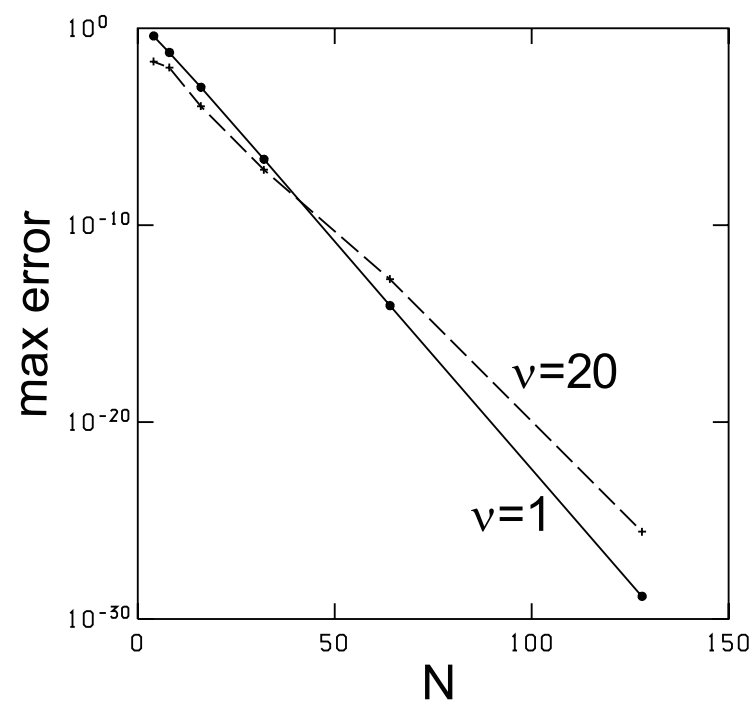

Fig. 4. The maximum error of Example 4. 
function of $N$ in Fig. 4 for $\nu=1$ and $\nu=20$. We again see high efficiency of this method via the integral equation.

If we compare the error graph for $\nu=20$ in Fig. 4 with that in Fig. 3 we see that the accuracy of the result presented in Fig. 4 is much better than that given in Fig. 3 with the method of Green's function. On the other hand, for $\nu=1$ both graphs almost overlap with each other. While the method of Green's function presented in Section 4 does not require solving a linear system of algebraic equations, the method via the integral equation requires solving one. In this respect, if the Green's function corresponding to the entire operator $L[u]$ of (1.1) is available the method of Green's function incorporated with the DE transformation is recommended, in particular for mild problems.

\section{References}

[1] R. Courant and D. Hilbert, Methods of Mathematical Physics (Vol.1). Interscience Publishers, INC., New York, 1937/1953, p.351.

[ 2 ] H.B. Keller, Numerical Methods for Two-Point Boundary-Value Problem. Blaisdel, 1968, Chapter 4.

[ 3 ] M. Muhammad and M. Mori, Double exponential formulas for numerical indefinite integration. J. Comput. Appl. Math., 161 (2003), 431-448.

[4] M. Muhammad, A. Nurmuhammad, M. Mori and M. Sugihara, Numerical solution of integral equations by means of the Sinc collocation method based on the double exponential transformation. J. Comput. Appl. Math., 177 (2005), 269-286.

[ 5 ] A. Nurmuhammad, M. Muhammad, M. Mori and M. Sugihara, Double exponential transformation in the Sinc-collocation method for the boundary value problem of fourth-order ordinary differential equation. J. Comput. Appl. Math., 182 (2005), 32-50.

[6] F. Stenger, Numerical Methods Based on Sinc and Analytic Functions. Springer, Berlin, New York, 1993.

[ 7 ] J. Stoer and R. Bulirsch, Introduction to Numerical Analysis. Translated by R. Bartels, et al., Springer-Verlag, New York, Tokyo, 1993.

[8] M. Sugihara, Double exponential transformation in the Sinc-collocation method for twopoint boundary value problems. J. Comput. Appl. Math., 149 (2002), 239-250.

[ 9 ] H. Takahasi, Complex function theory and numerical analysis. Publ. RIMS Kyoto Univ., 41 (2005), 979-988.

[10] H. Takahasi and M. Mori, Double exponential formula for numerical integration. Publ. RIMS Kyoto Univ., 9 (1974), 721-741.

[11] K. Tanaka, M. Sugihara and K. Murota, Numerical indefinite integration by double exponential sinc method. Math. Comp., 74 (2004), 655-679. 